\title{
Arthroscopic Viewing Position Affects Anterior Cruciate Ligament Reconstruction Femoral Tunnel Length Measurements
}

\author{
Sheeba M. Joseph ${ }^{1}$, Michael R. Karns ${ }^{1,2}$, Derrick M. Knapik ${ }^{1,2 *}$ and James E. Voos ${ }^{1,2}$ \\ ${ }^{1}$ Department of Orthopaedic Surgery, University Hospitals Cleveland Medical Center, Cleveland, OH, United States, ${ }^{2}$ Sports \\ Medicine Institute, University Hospitals Cleveland Medical Center, Cleveland, OH, United States
}

\section{OPEN ACCESS}

Edited by:

Vassilios S. Nikolaou, University General Hospital Attikon, Greece

Reviewed by: Michael-Alexander Malachias, National and Kapodistrian University of Athens, Greece Leonidas Roumeliotis, National and Kapodistrian University of Athens, Greece

*Correspondence:

Derrick M. Knapik derrick.knapik@gmail.com

Specialty section: This article was submitted to Orthopedic Surgery,

a section of the journal

Frontiers in Surgery

Received: 08 December 2017 Accepted: 12 February 2018 Published: 01 March 2018

Citation: Joseph SM, Karns MR, Knapik DM and Voos JE (2018) Arthroscopic Viewing Position Affects Anterior Cruciate Ligament Reconstruction Femoral Tunnel Length Measurements.

Front. Surg. 5:16.

doi: 10.3389/fsurg.2018.00016
Purpose: To purpose of this study was to compare arthroscopic anterior cruciate ligament (ACL) reconstruction femoral tunnel length measurements from the anterolateral portal between the standard notch view using a $30^{\circ}$ arthroscope versus a "top-down" view utilizing a $70^{\circ}$ arthroscope to visual the far side of the femoral tunnel aperture.

Methods: Arthroscopic femoral tunnel length measurements using calibrated reamers from the standard notch versus the "top-down" view were obtained and reviewed in 54 skeletally mature patients undergoing $A C L$ reconstruction with no prior bony knee surgery. Patient age, height, weight, sex, and surgery laterality were also recorded. Measurements of femoral tunnel length were repeated using both views for interobserver and intra-observer correlation.

Results: Inter-observer and intra-observer intra-class correlation coefficients for the standard notch view and "top-down" views were excellent, with higher reliability values appreciated using the "top down" view. Mean overall femoral tunnel length measurements obtained using the standard notch view were significantly longer than measurements from the "top-down" view $(p<0.001)$.

Conclusions: The standard notch view provides significantly longer femoral tunnel length measurements in comparison to the "top-down" view.

Keywords: ACL reconstruction, arthroscopy, femoral tunnel length, seventy-degree arthroscopy, graft-tunnel mismatch

\section{INTRODUCTION}

As of 2015, over 200,000 anterior cruciate ligament (ACL) reconstructions are performed each year in the United States.(1-3) To improve patient outcomes and return to play rates, advances in surgical techniques have sought to achieve more anatomic reconstructions.(4) As such, multiple techniques have been developed to enhance the surgeon's ability to create the femoral tunnel at the anatomic origin of the ACL including drilling from an accessory medial portal with the knee in a hyper-flexed $\left(\sim 120^{\circ}\right)$ position. $(5,6)$ However, drilling the femoral tunnel at such a low, posterior position in the hyper-flexed knee using rigid reamers increases the risks for short tunnels, posterior cortical blowout, and common peroneal nerve injury if the guide pins exits inferior to the biceps femoris. (7-10) Several commercially available flexible reamer systems have been developed permitting drilling of an anatomically placed femoral tunnel closer to $90^{\circ}$ of knee 
flexion, resulting in longer tunnel lengths and safer distances from the common peroneal nerve. $(11,12)$

Achieving anatomic femoral tunnel placement and subsequent graft insertion demands adequate visualization of the intercondylar notch wall of the lateral femoral condyle. Osaki et al examined the femoral tunnel aperture within the intercondylar notch and demonstrated a discrepancies of up to $5 \mathrm{~mm}$ using an outside-in drilling technique and $4.2 \mathrm{~mm}$ with a trans-portal drilling technique between tunnel lengths measured at the center versus the shortest aspects of the femoral tunnel aperture.(13) The authors attributed their findings to the obliquity of the femoral tunnel relative to the lateral femoral intercondylar notch wall. Accordingly, the anatomy and inevitable oblique orientation for femoral tunnel drilling may result in overestimation of tunnel length if the near side (i.e., distal aspect) of the aperture is referenced versus referencing the far side (i.e., proximal aspect) of the aperture. Such tunnel length inaccuracy may lead to a proud plug with use of a bonetendon-bone graft or an insufficient graft within the tunnel using soft-tissue only grafts.(14)

Visualizing the entire femoral ACL footprint can be challenging from a standard anterolateral-viewing portal using a $30^{\circ}$ arthroscope. Several authors have described improved visualization of the femoral ACL footprint using a $70^{\circ}$ arthroscope from the lateral portal. $(14,15)$ Moreover, use of the $70^{\circ}$ arthroscope using the standard anterolateral viewing portal enables the surgeons to achieve a "top-down" view of the femoral reamer when using the trans-portal technique for drilling the femoral tunnel, serving as the senior author's preferred technique during ACL reconstruction. Unlike the standard notch view obtained with a $30^{\circ}$ arthroscope from the anterolateral portal, this "top-down" view permits visualization of the far side (i.e., proximal aspect) of the ACL femoral tunnel aperture. The purpose of the study is to compare femoral tunnel length measurements using the "topdown" view with a $70^{\circ}$ arthroscope to reference the far side of the femoral tunnel aperture versus measurements from the standard notch view using a $30^{\circ}$ arthroscope to reference the near side. The authors hypothesized that femoral tunnel length measurements obtained from the "top-down" view would be more accurate than measurements obtained using the standard notch view.

\section{METHODS}

The study protocol was pre-approved by the author's Institutional Review Board. Arthroscopic images of skeletally mature patients with closed physes undergoing primary ACL reconstruction by a single fellowship-trained Orthopaedic sports surgeon between July 1, 2014 and December 31, 2016 were retrospectively reviewed for study inclusion. As such, no formal patient consent was required. Skeletally immature patients and those with prior bony knee surgery were excluded. Demographic data including patient age, sex, height, weight and laterality of surgery were recorded.

\section{Surgical Technique}

A standard $30^{\circ}$ arthroscope was used for the diagnostic arthroscopy and to address any chondral or meniscal pathology.
It is the senior author's preference to use a $70^{\circ}$ arthroscope for the ACL reconstruction portion of the operation to view the intercondylar notch and ACL footprint, perform tunnel drilling, and visualize graft insertion. At the time of femoral tunnel drilling using a flexible reamer (Clancy Flexible Reamer, Smith and Nephew, Memphis, TN) the $70^{\circ}$ arthroscope is placed in the standard anterolateral portal looking laterally at the reamer. The surgeon stopped the reamer and obtained the first of two images of the reamer within the tunnel. Despite using a $70^{\circ}$ arthroscope, the image obtained is identical to that obtained with a $30^{\circ}$ arthroscope given then positioning of the light cord/ camera lens. (Figure 1A,B) Care was taken to ensure a hash mark number label was clearly visible on the reamer in the image as reference for measurements of tunnel depth. The second image was obtained by rotating the camera $90^{\circ}$ to achieve the "topdown" view, which orients the visual field looking down on the reamer to visualize the far side (i.e., back-side) of the reamer and femoral tunnel aperture. (Figure 1C,D) Care was again taken to ensure inclusion of a hash mark number label on the reamer for reference of tunnel depth.

\section{Measurements and Calculations}

The flexible reamer's links between each $5 \mathrm{~mm}$ labeled hash mark were measured using a digital caliper with accuracy to $0.01 \mathrm{~mm}$ (Mitutoyo, Mitutoyo Corp., Model Japan). A single metal link was measured to be $1.01 \mathrm{~mm}$ long. (Figure 2A) The length of a single link and the two adjacent gaps on either side was measured as 1.89 $\mathrm{mm}$ long. (Figure 2B) These two measurements were rounded to the nearest $0.1 \mathrm{~mm}$. Thus, the length of the space between two links was determined by subtracting the length of a single link $(1.0 \mathrm{~mm})$ and then dividing the remaining length by two, given the presence of two inter-link gaps. [i.e., $(1.9-1.0 \mathrm{~mm}) / 2=0.45 \mathrm{~mm}$ ]. (Figure $2 \mathrm{C}$ ) Thus, the femoral tunnel length could be accurately calculated from arthroscopic images by referencing the visualized hash mark number label and subtracting the link lengths $(1.0 \mathrm{~mm})$ and the inter-link gap lengths $(0.45 \mathrm{~mm})$ until the point at which the reamer intersected with the femoral tunnel wall aperture. The femoral tunnel length measurements were determined from the surgical arthroscopic images using the standard notch view and the "top-down" view for each patient.

\section{Statistical Analysis}

An a priori power analysis was conduced to determine the minimum number of patients necessary to detect a significant difference in femoral tunnel length measurements between views. Using an initial set of 20 measurements ( $n=10$ patients), SD was assumed to be $0.63 \mathrm{~mm}$. With an alpha-level of 0.05 and power set to $95 \%$, the minimum number of patients needed to detect a $0.5 \mathrm{~mm}$ difference was calculated to be 54 patients.(16)

All data were analyzed for normality using the Shapiro-Wilk test. An intraclass correlation coefficient (ICC) for femoral tunnel length measurements was calculated by the senior author at two separate time points (minimum: 2 weeks apart) in all patients ( $n=54$ patients; $n=108$ images) while an interclass class correlation coefficient was calculated in 26 patients ( $n=52$ images) between two authors. Following established recommendations, 

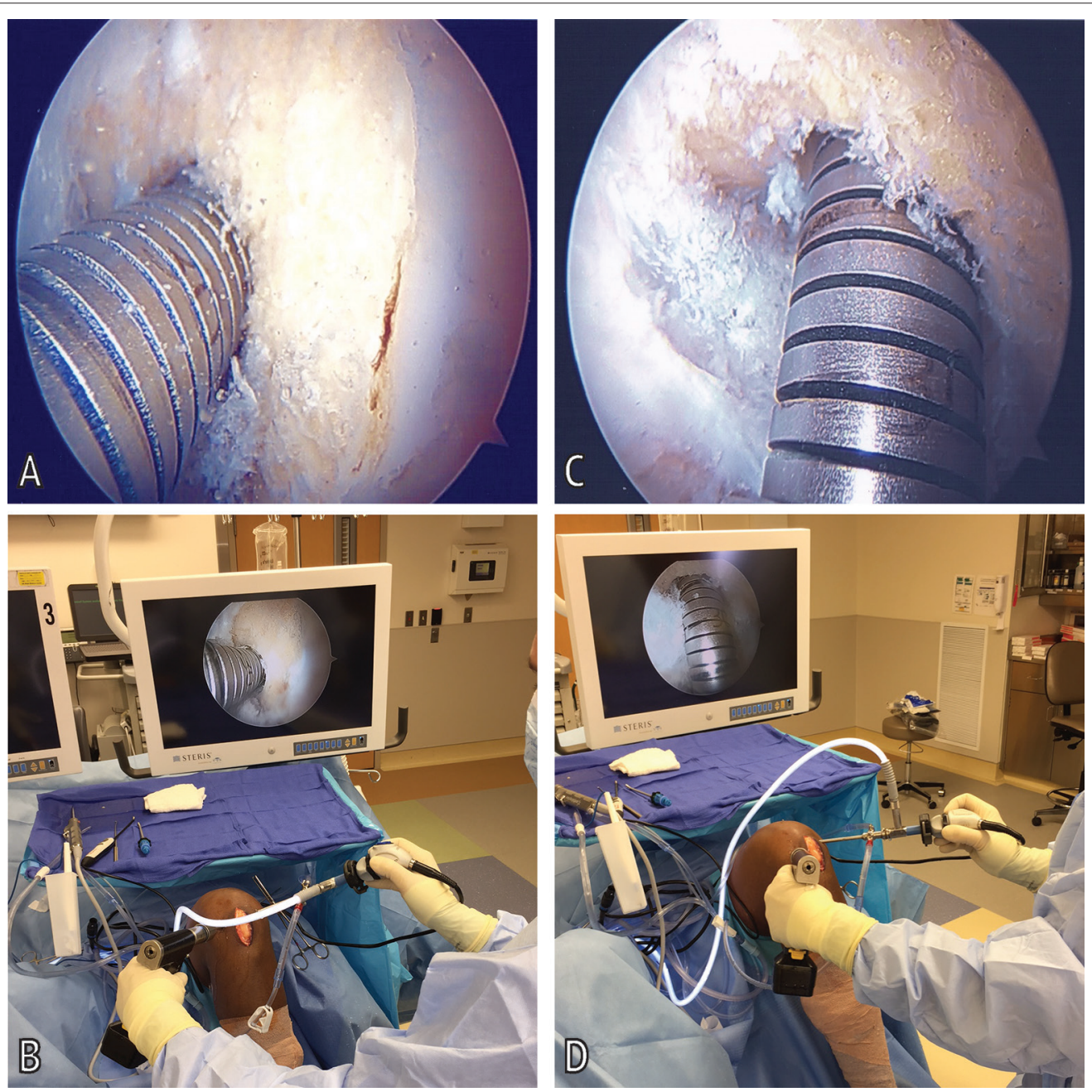

FIGURE 1 | Arthroscopic images of a left knee during femoral tunnel reaming to obtain femoral tunnel reamer measurements. (A) Standard notch view (B) Surgeon hand and camera position for obtaining standard notch view (C) Top-down view (D) Surgeon hand and camera position for obtaining top-down view.

an ICC of $<0.4$ was determined to be poor, $0.4-0.75$ to be fair to good, and $>0.75$ to be excellent. $(17,18)$ Paired samples $t$-test was used to test the null hypothesis that no significant difference would be present between measurements obtained via the standard notch view versus measurements obtained via the "top-down" view. An independent sample $t$-test was used to compare dichotomous variables (patient sex, laterality of surgery) in paired measurements obtained from the two views. Pearson's correlations were used to compare age, height, and weight to the difference in paired measurements obtained from the two views. A $p$-value of $<0.05$ was used to determine statistical significance. All statistical analysis was performed using SPSS (Version 23, IBM, Armonk, New York) software.

\section{RESULTS}

A total of 60 patients underwent ACL reconstruction during the study period with imaging and documentation of both standard-notch and "top-down" views for femoral tunnel length measurement. Six patients were excluded due to bony debris obscuring the reamer's hash mark number labels, preventing accurate tunnel length measurement in at least one of the views. Mean age of the 54 patients included for final analysis was $26 \pm 9.8$ years (range, 15 to 51 years) with a mean height and weight of $69 \pm 4.4$ inches (range, 58-77 inches) and $84.0 \pm 18.7$ kilograms (range, 54.0-136 kilograms), respectively. The study group included 19 females and 35 males. Twenty-seven patients underwent surgery to the left knee while 27 had surgery on the right knee.

Measurements of both the standard notch view and "top down" view produced excellent intra-observer $(0.0877,0.932$; respectively) and inter-observer $(0.916,0.960$; respectively) values. Femoral tunnel length measurements obtained via the standard notch view were significantly longer than those obtained via the "top-down" view $(p<0.001$; mean $2.24 \pm 0.868$ $\mathrm{mm}$ longer) (Figures 3 and 4). Univariate analysis showed that the difference in femoral tunnel length measurements obtained via the standard notch-view versus the top-down view did not 

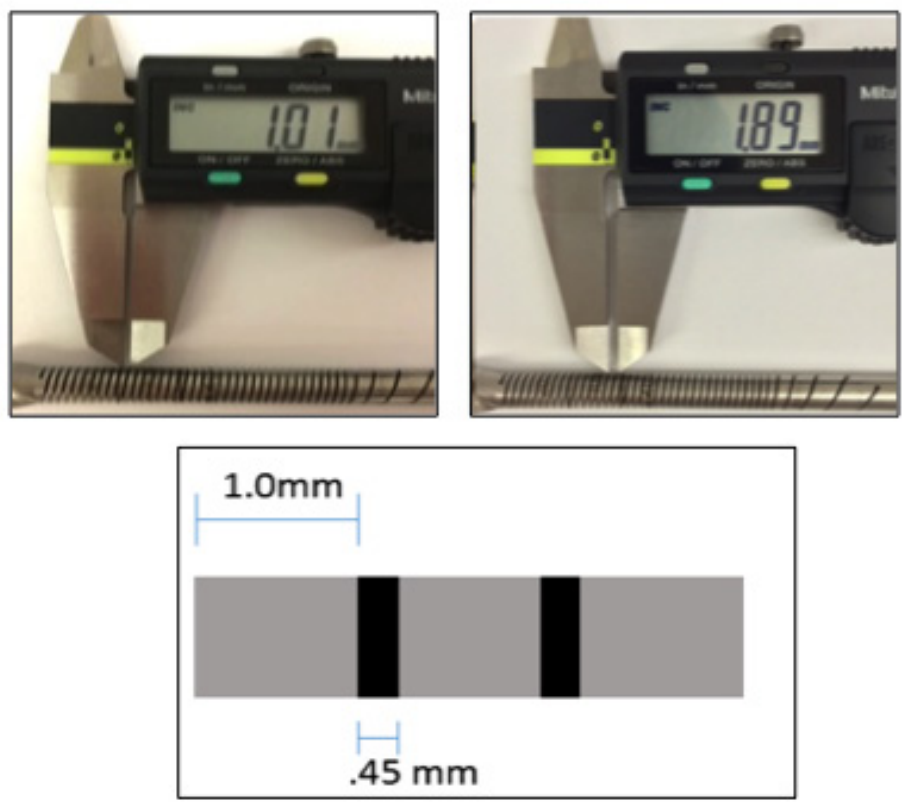

FIGURE 2 | Digital caliper measurement of flexible reamer links and gaps for femoral tunnel length measurements.

vary significantly with age $(\mathrm{r}=0.12, p=0.40)$, height $(\mathrm{r}=0.013$, $p=0.92)$, or weight $(r=0.042, p=0.76)$. There was no significant difference in mean femoral tunnel length measurements between females (mean, $2.27 \pm 0.666 \mathrm{~mm}$ ) and males (mean, $2.22 \pm 0.974 \mathrm{~mm})(p=0.852)$. Furthermore, no significant difference in femoral tunnel length measurements was appreciated based on surgical laterality (mean, $2.08 \pm 0.776 \mathrm{~mm}$ for left versus $2.40 \pm 0.94457 \mathrm{~mm}$ for right; $p=0.179$ ).

\section{DISCUSSION}

The purpose of this investigation was to better understand different in femoral tunnel length measurements during ACL reconstruction comparing the standard notch view using a $30^{\circ}$ arthroscope versus the "top down" view with a $70^{\circ}$ arthroscope. The authors found that in 54 subjects undergoing ACL reconstruction, measurements of femoral tunnel length via the standard notch view were significantly longer than those obtained using a "top down" view. Patient sex, age, height, weight and surgical laterality had no significant impact on mean femoral tunnel length measurements. Data from this investigation adds to the growing body of research regarding femoral tunnel aperture morphology and its clinical implications during ACL reconstruction.(13, 19, 20)

Multiple studies have implicated non-anatomic femoral tunnel placement as the reason for ACL reconstruction failure and instability post-operatively $(21-23)$ as current efforts towards achieving optimal stability and outcomes have focused on anatomic ACL reconstruction techniques. $(4,24)$ Obtaining anatomic femoral tunnel placement and subsequent tunnel length measurement relies on adequate visualization of the native ACL footprint. Numerous techniques have been proposed for gaining better visualization of the lateral femoral condylar wall within the notch, including a modified mid-patellar portal (25) and a central accessory medial portal.(26) By using a $70^{\circ}$ arthroscope through the standard anterolateral portal, the "topdown" view described in this study provides the surgeon with an unobstructed view of the far side of the femoral tunnel aperture without the need for extra portals. By providing a perpendicular 


\section{Femoral Tunnel Length Measurements Comparison}

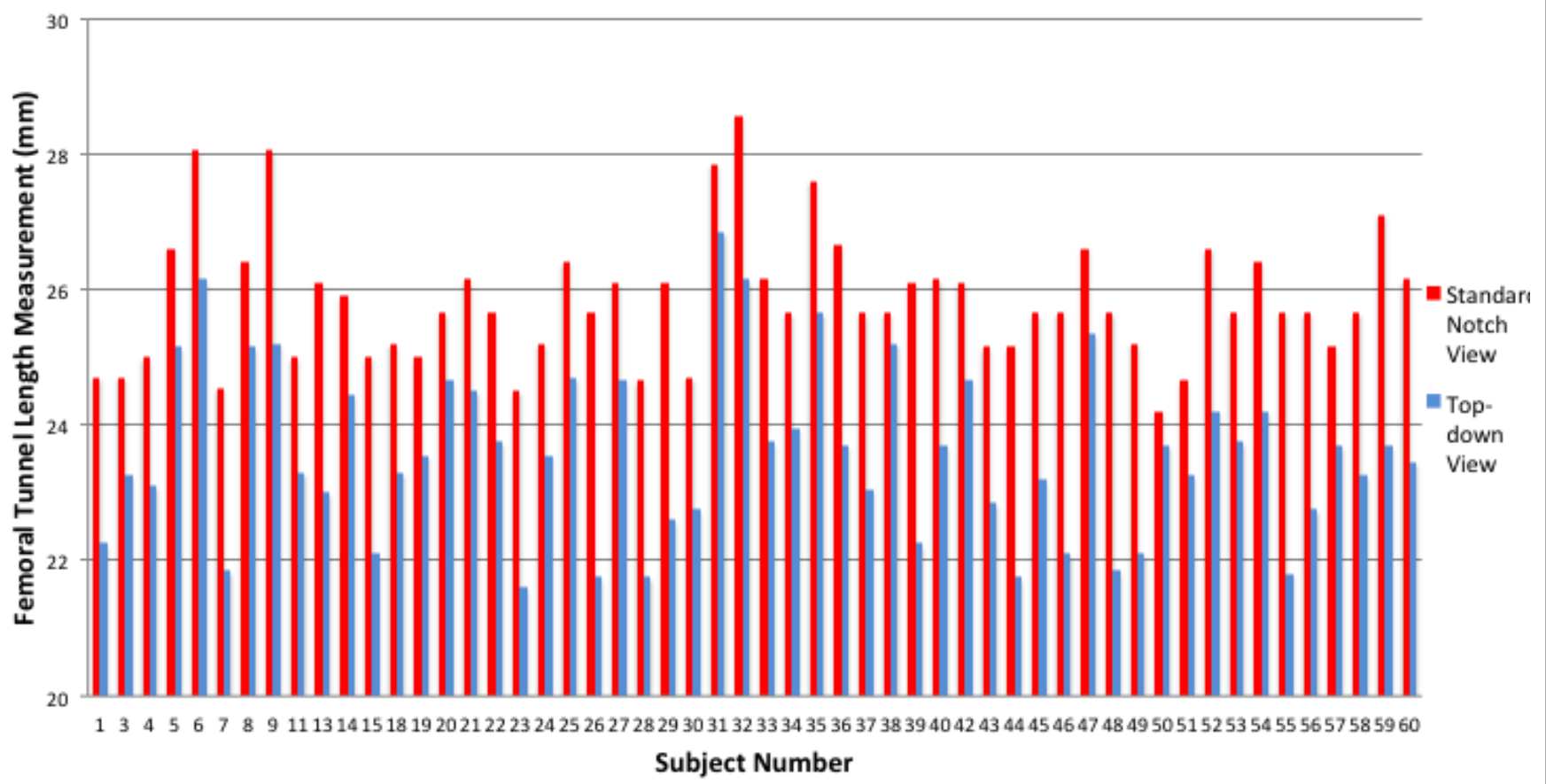

Subject Number

FIGURE 3 | Comparison of femoral tunnel length measurements obtained by the standard notch view and the top-down view.

view of the reamer at the femoral tunnel aperture, the authors found excellent intra- and inter-observer reliability values, indicative of the high reproducibility of measurements using the "top down" view. In their investigation utilizing a 3-dimensional knee model, Hoshino et al. further demonstrated the ability to reduce distortion and consequent inaccurate measurement readings using the $70^{\circ}$ arthroscope when compared views obtained using $0^{\circ}$ and $30^{\circ}$ arthroscopes.(27)

Compared to the standard notch view, the "top down" view avoids over-estimation of femoral tunnel length measurements. This effectively mitigates potential complications associated with graft-tunnel length mismatch, which has been shown to be crucial in order to ensure optimal outcomes following ACL reconstruction. $(28,29)$ When using a bone-patellar tendon-bone graft or osseous grafts such as an Achilles or quadriceps tendon, over-estimation of the femoral tunnel length may lead to a proud bone plug within the notch and/or inadequate bone-plug interface at the tibial tunnel, compromising the integrity of interference screw fixation (30), leading to intra-operative or postoperative complications.(31) Arguably, a buffer distance may be built into graft length and tunnel drilling calculations. However, having a femoral bone plug that ends flush with the femoral tunnel aperture within the notch avoids potential complications associated with a recessed femoral bone plug such as the "windshield wiper effect," thought to result in tunnel widening and graft abrasion. $(32,33)$

Moreover, for a soft-tissue graft, overestimation of femoral tunnel length can lead to placement of an inadequate amount of the graft within the femoral tunnel.(34) Lee et al. demonstrated that such grafts incompletely fill femoral tunnel apertures and tend to rest off-center within the tunnel.(35) Accordingly, a discordant measurement of osseous tunnel length versus graft length may be amplified with the graft's final eccentric resting position. Previous investigations have examined the temporal changes in the cross-sectional area of the femoral tunnel aperture, enlarging with time (36) with slower remodeling of the bone-tendon interface at the intra-articular aperture.(37) The consequences of these natural history changes in ACL 


\section{Arthroscopic Femoral Tunnel Length Measurements}

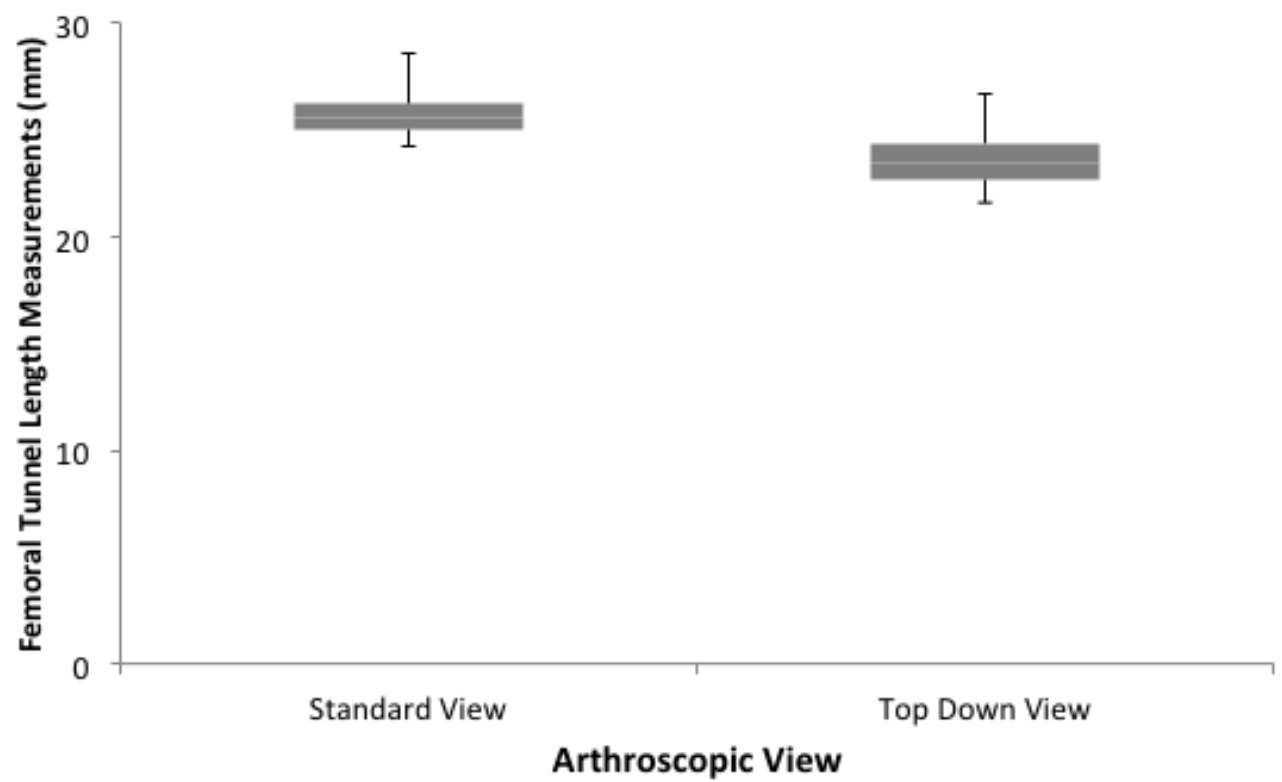

FIGURE 4 | Box plots of femoral tunnel length measurements in millimeters by arthroscopic technique. Upper and lower hinges represent 25 and $75 \%$ quartiles; middle represent median or $50 \%$ quartile.

reconstruction grafts are magnified with increased motion at the tunnel aperture, resulting in lower rates of healing at sites of motion.(38)

This study was not without limitations. A single surgeon obtained all arthroscopic photographs used to measure femoral tunnel length during surgery. While use of the $70^{\circ}$ arthroscope is the senior author's preference, inviting the potential for observer bias, the excellent inter-observer reliability values obtained demonstrate the validity of the methods utilized and results obtained. However, additional studies utilizing multiple different surgeons is warranted to further validate the conclusions and surgical technique of the "top down" view. Second, if suspensory fixation techniques with an adjustable loop fixation function are utilized, then concerns about graft-tunnel mismatch become less pertinent since the loop portion of the graft-button construct is adjustable following passage of the graft.(20) Lastly, post-operative functional outcome scores and complications rates were not analyzed, as such despite the reported significant difference in femoral tunnel length overestimation using the standard notch view, the clinical relevance of this finding cannot be extrapolated and is beyond the scope of this investigation.

\section{CONCLUSIONS}

The "top-down" view using a $70^{\circ}$ arthroscope provides a viable alternative to the standard notch view with a $30^{\circ}$ arthroscope for visualizing the anatomic ACL footprint. The "top down" view helps avoid tunnel length overestimation by referencing the backside of the reamer for tunnel length measurements, especially in cases where lateral femoral intercondylar notch wall obliquity is high or the tunnel drilling obliquity is high. By providing a more accurate assessment of femoral tunnel length, the surgeon can plan appropriately for final tunnel length preparation and graft length preparation, facilitating more advantageous ACL reconstruction by avoiding complications associated with grafttunnel length mismatch. Future long-term prospective studies 
examining functional outcomes and complication rates following ACL reconstruction utilizing the "top-down" view with a $70^{\circ}$ arthroscope versus the standard notch view with a $30^{\circ}$ arthroscope are necessary to better understand the clinical impact of technique on outcomes following reconstruction.

\section{ETHICS STATEMENT}

This study was carried out in accordance with the recommendations of the University Hospitals Cleveland Institutional Review Board. The protocol was approved by the University Hospitals Institutional

\section{REFERENCES}

1. Buller LT, Best MJ, Baraga MG, Kaplan LD. Trends in anterior cruciate ligament reconstruction in the United States. Orthop J Sports Med (2015) 3(1):2325967114563664):. doi: 10.1177/2325967114563664

2. Evans CL, Miller MD, Diduch DR. Revision anterior cruciate ligament surgery: one-stage versus two-stage technique. Insall \& Scott: Surgery of the Knee. Vol. 1. 6th ed. Elsevier (2018). p. 685-701.

3. Mall NA, Chalmers PN, Moric M, Tanaka MJ, Cole BJ, Bach BR, et al. Incidence and trends of anterior cruciate ligament reconstruction in the United States. Am J Sports Med (2014) 42(10):2363-70. doi: $10.1177 / 0363546514542796$

4. Dhawan A, Gallo RA, Lynch SA. Anatomic tunnel placement in anterior cruciate ligament reconstruction. J Am Acad Orthop Surg (2016) 24(7):443-54. doi: 10.5435/JAAOS-D-14-00465

5. Harner CD, Honkamp NJ, Ranawat AS. Anteromedial portal technique for creating the anterior cruciate ligament femoral tunnel. Arthroscopy (2008) 24(1):113-5. doi: 10.1016/j.arthro.2007.07.019

6. Tompkins M, Milewski MD, Brockmeier SF, Gaskin CM, Hart JM, Miller MD. Anatomic femoral tunnel drilling in anterior cruciate ligament reconstruction: use of an accessory medial portal versus traditional transtibial drilling. Am J Sports Med (2012) 40(6):1313-21. doi: 10.1177/0363546512443047

7. Bedi A, Raphael B, Maderazo A, Pavlov H, Williams RJ. Transtibial versus anteromedial portal drilling for anterior cruciate ligament reconstruction: a cadaveric study of femoral tunnel length and obliquity. Arthroscopy (2010) 26(3):342-50. doi: 10.1016/j.arthro.2009.12.006

8. Lee DH, Kim HJ, Ahn HS, Bin SI. Comparison of femoral tunnel length and obliquity between transtibial, anteromedial portal, and outside-in surgical techniques in single-bundle anterior cruciate ligament reconstruction: a meta-analysis. Arthroscopy (2016) 32(1):142-50. doi: 10.1016/j. arthro.2015.07.026

9. Lubowitz JH. Anteromedial portal technique for the anterior cruciate ligament femoral socket: pitfalls and solutions. Arthroscopy (2009) 25(1):95-101. doi: 10.1016/j.arthro.2008.10.012

10. Lubowitz JH, Konicek J. Anterior cruciate ligament femoral tunnel length: cadaveric analysis comparing anteromedial portal versus outside-in technique. Arthroscopy (2010) 26(10):1357-62. doi: 10.1016/j. arthro.2010.02.014

11. Fitzgerald J, Saluan P, Richter DL, Huff N, Schenck RC. Anterior cruciate ligament reconstruction using a flexible reamer system: technique and pitfalls. Orthop J Sports Med (2015) 3(7):2325967115592875. doi: $10.1177 / 2325967115592875$

12. Silver AG, Kaar SG, Grisell MK, Reagan JM, Farrow LD. Comparison between rigid and flexible systems for drilling the femoral tunnel through an anteromedial portal in anterior cruciate ligament reconstruction. Arthroscopy (2010) 26(6):790-5. doi: 10.1016/j.arthro.2009.10.012

13. Osaki K, Okazaki K, Matsubara H, Kuwashima U, Murakami K, Iwamoto Y. Asymmetry in femoral tunnel socket length during anterior cruciate ligament reconstruction with transportal, outside-in, and modified
Review Board who deemed that written consent from patients was not required due to the retrospective nature of the investigation.

\section{AUTHOR CONTRIBUTIONS}

SMJ: project conception and design, measurements, data analysis, manuscript preparation. MRK: project conception and design, measurements, data analysis. DMK: data analysis and manuscript preparation. JEV: project conception and design, obtained surgical images, manuscript preparation. transtibial techniques. Arthroscopy (2015) 31(12):2365-70. doi: 10.1016/j. arthro.2015.06.026

14. Bucher TA, Naim S, Mandalia V. The use of the $70^{\circ}$ arthroscope for anatomic femoral and tibial tunnel placement and tunnel viewing in anterior cruciate ligament reconstruction. Arthrosc Tech (2014) 3(1):e79-81. doi: 10.1016/j. eats.2013.08.001

15. Bedi A, Dines J, Dines DM, Kelly BT, O'Brien SJ, Altchek DW, et al. Use of the $70^{\circ}$ arthroscope for improved visualization with common arthroscopic procedures. Arthroscopy (2010) 26(12):1684-96. doi: 10.1016/j.arthro.2010.04.070

16. Faul F, Erdfelder E, Lang AG, Buchner A. G*Power 3: a flexible statistical power analysis program for the social, behavioral, and biomedical sciences. Behav Res Methods (2007) 39(2):175-91. doi: 10.3758/BF03193146

17. Fleiss J. The design and analysis of clinical experiments. New York: Wiley (1986).

18. Shrout PE, Fleiss JL. Intraclass correlations: uses in assessing rater reliability. Psychol Bull (1979) 86(2):420-8. doi: 10.1037/0033-2909.86.2.420

19. Hensler D, Working ZM, Illingworth KD, Thorhauer ED, Tashman S, Fu FH. Medial portal drilling: effects on the femoral tunnel aperture morphology during anterior cruciate ligament reconstruction. J Bone Joint Surg Am (2011) 93(22):2063-71. doi: 10.2106/JBJS.J.01705

20. Okazaki K, Osaki K, Nishikawa K, Matsubara H, Tashiro Y, Iwamoto Y. Overestimation of femoral tunnel length during anterior cruciate ligament reconstruction using the retrograde outside-in drilling technique. Arch Orthop Trauma Surg (2016) 136(8):1159-63. doi: 10.1007/s00402-016-2492-y

21. Wright RW, Huston LJ, Spindler KP, Dunn WR, Haas AK, Allen CR, et al. Descriptive epidemiology of the Multicenter ACL Revision Study (MARS) cohort. Am J Sports Med (2010) 38(10):1979-86. doi: 10.1177/0363546510378645

22. Morgan JA, Dahm D, Levy B,Stuart MJ MARS Study Group. Femoral tunnel malposition in ACL revision reconstruction. J Knee Surg (2012) 25(5):361-8. doi: $10.1055 / \mathrm{s}-0031-1299662$

23. Samitier G, Marcano AI, Alentorn-Geli E, Cugat R, Farmer KW, Moser MW. Failure of anterior cruciate ligament reconstruction. Arch Bone Jt Surg (2015) $3(4): 220-40$

24. Pinczewski LA, Salmon LJ, Jackson WF, von Bormann RB, Haslam PG, Tashiro S. Radiological landmarks for placement of the tunnels in single-bundle reconstruction of the anterior cruciate ligament. J Bone Joint Surg Br (2008) 90(2):172-9. doi: 10.1302/0301-620X.90B2.20104

25. Calvisi V, Lupparelli S, Giuliani P. A view from above: a modified Patel's medial midpatellar portal for anterior cruciate ligament arthroscopic surgery. Arthroscopy (2007) 23(3):324.e1-5. doi: 10.1016/j.arthro.2006.07.040

26. Cohen SB, Fu FH. Three-portal technique for anterior cruciate ligament reconstruction: use of a central medial portal. Arthroscopy (2007) 23(3):325. e1-5. doi: 10.1016/j.arthro.2006.07.030

27. Hoshino Y, Rothrauff BB, Hensler D, Fu FH, Musahl V. Arthroscopic image distortion-part II: the effect of lens angle and portal location in a 3D knee model. Knee Surg Sports Traumatol Arthrosc (2016) 24(6):2072-8. doi: 10.1007/ s00167-014-3268-y

28. Grawe B, Smerina A, Allen A. Avoiding graft-tunnel length mismatch in anterior cruciate ligament reconstruction: the single-bone plug technique. Arthrosc Tech (2014) 3(3):e417-20. doi: 10.1016/j.eats.2014.04.003 
29. Kopf S, Forsythe B, Wong AK, Tashman S, Anderst W, Irrgang JJ, et al. Nonanatomic tunnel position in traditional transtibial single-bundle anterior cruciate ligament reconstruction evaluated by three-dimensional computed tomography. J Bone Joint Surg Am (2010) 92(6):1427-31. doi: 10.2106/ JBJS.I.00655

30. Brown JA, Brophy RH, Franco J, Marquand A, Solomon TC, Watanabe D, et al. Avoiding allograft length mismatch during anterior cruciate ligament reconstruction: patient height as an indicator of appropriate graft length. Am J Sports Med (2007) 35(6):986-9. doi: 10.1177/0363546506298584

31. Yanke A, Ellman MB, Sherman SL, Bach BR. Graft-tunnel mismatch in bonetendon-bone ACL reconstruction: preventation and treatment. Tech Orthop (2012) 27(2):153-7.

32. Gill TJ, Steadman JR. Anterior cruciate ligament reconstruction the twoincision technique. Orthop Clin North Am (2002) 33(4):727-35.

33. Yanke A, Ellman MB, Sherman SL, Bach BRJ. Graft-tunnel mismatch in bonetendon-bone ACL reconstruction: techniques in orthopaedics. Tech Orthop (2012) 27(2):153-7.

34. Zantop T, Ferretti M, Bell KM, Brucker PU, Gilbertson L, Fu FH. Effect of tunnel-graft length on the biomechanics of anterior cruciate ligamentreconstructed knees: intra-articular study in a goat model. Am J Sports Med (2008) 36(11):2158-66. doi: 10.1177/0363546508320572

35. Lee BH, Bansal S, Park SH, Wang JH. Eccentric graft positioning within the femoral tunnel aperture in anatomic double-bundle anterior cruciate ligament reconstruction using the transportal and outside-in techniques. Am J Sports Med (2015) 43(5):1180-8. doi: 10.1177/0363546514568278

36. Tachibana Y, Mae T, Shino K, Kanamoto T, Sugamoto K, Yoshikawa H, et al. Morphological changes in femoral tunnels after anatomic anterior cruciate ligament reconstruction. Knee Surg Sports Traumatol Arthrosc (2015) 23(12):3591-600. doi: 10.1007/s00167-014-3252-6

37. Bedi A, Kawamura S, Ying L, Rodeo SA. Differences in tendon graft healing between the intra-articular and extra-articular ends of a bone tunnel. Hss $J$ (2009) 5(1):51-7. doi: 10.1007/s11420-008-9096-1

38. Rodeo SA, Kawamura S, Kim HJ, Dynybil C, Ying L. Tendon healing in a bone tunnel differs at the tunnel entrance versus the tunnel exit: an effect of graft-tunnel motion? Am J Sports Med (2006) 34(11):1790-800. doi: $10.1177 / 0363546506290059$

Conflict of Interest Statement: The authors declare that the research was conducted in the absence of any commercial or financial relationships that could be construed as a potential conflict of interest.

Copyright (c) 2018 Joseph, Karns, Knapik and Voos. This is an open-access article distributed under the terms of the Creative Commons Attribution License (CC BY). The use, distribution or reproduction in other forums is permitted, provided the original author(s) and the copyright owner are credited and that the original publication in this journal is cited, in accordance with accepted academic practice. No use, distribution or reproduction is permitted which does not comply with these terms. 